\title{
New thermometer and oxygen fugacity sensor calibrations for ilmenite- and chromian spinel-bearing peridotitic assemblages
}

Taylor, W.R. ${ }^{1}$, Kamperman, M. ${ }^{2}$, and Hamilton, R. ${ }^{3}$

${ }^{1}$ R.S.E.S., Australian National University, Canberra, A.C.T. 0200, Australia

2BHP Iron Ore, Newman, W.A. 6753, Australia (formerly Geology Dept, University of Tasmania).

${ }^{3}$ Southstar Resources NL, 50 Colin St, West Perth, W.A. 6005, Australia (formerly WMC, Perth).

New Fe-Mg exhange thermometers and oxygen sensors have been developed for high pressure peridotitic assemblages that include ilmenite and/or chromian spinel. The calibrations are based on new experimental work to $3.5 \mathrm{GPa}$ designed to investigate $\mathrm{fO}_{2}$ and temperature sensitive equilibria in which the effects of $\mathrm{Cr}_{2} \mathrm{O}_{3}$ (eskolaite) substitution in ilmenite and $\mathrm{Ti}$ substitution in chromian spinel have been specifically considered. These substitutions are important in mantle-derived ilmenite and chromite solid solutions, particularly those associated with diamond-facies peridotite. The results show that eskolaite substitution in ilmenite has a significant effect on olivine-ilmenite Fe-Mg temperature, in fact, compared to the new calibration, older calibrations yield $\mathrm{T}$ differences of up to several hundred ${ }^{\circ} \mathrm{C}$. These results suggest that $\mathrm{Cr}$-rich ilmenites are likely to be of higher $\mathrm{P}, \mathrm{T}$ origin than previously assumed. For spinel-bearing assemblages, the effect of $\mathrm{Ti}$ substitution in spinel was found to have a relatively small effect on $\mathrm{fO}_{2}$ and $\mathrm{Fe}-\mathrm{Mg}$ temperature, however, the new olivine-spinel oxygen sensor and thermometer calibrations have resulted in an improved fit to existing experimental data. The new calibrations offer advances in defining the temperature and redox conditions favourable for diamond stability in the upper mantle and as such may be usefully applied to 'diamond-indicator' mineral and xenolith suites sampled by kimberlite and other rocks of deep-seated origin.

Olivine-ilmenite $\mathrm{Fe}-\mathrm{Mg}$ exchange thermometer :

$\begin{aligned} \mathrm{T}(\mathrm{K})= & {\left[-13715+\mathrm{P} \cdot \Delta \mathrm{V}+3785 \cdot\left(2 \mathrm{X}_{\mathrm{fa}}-1\right)+2830 \cdot\left(\mathrm{X}_{\mathrm{gk}}-\mathrm{X}_{\mathrm{ilm}}\right)-19560 \cdot \mathrm{X}_{\mathrm{hem}}\right.} \\ & \left.-7840 \cdot \mathrm{X}_{\mathrm{esk}}+45122 \cdot \mathrm{X}_{\mathrm{hem}} \cdot \mathrm{X}_{\mathrm{esk}}\right] /\left[2.231-\mathrm{R} \cdot \mathrm{ln} \mathrm{K}_{\mathrm{d}}\right]\end{aligned}$

$\Delta \mathrm{V}=0.011 \cdot\left(\mathrm{X}_{\mathrm{gk}}-\mathrm{X}_{\mathrm{ilm}}\right)-0.047+0.015 \cdot\left(2 \mathrm{X}_{\mathrm{fa}}-1\right)$

$\mathrm{K}_{\mathrm{d}}=\left[\mathrm{X}_{\mathrm{fo}} \cdot \mathrm{X}_{\mathrm{ilm}}\right] /\left[\mathrm{X}_{\mathrm{fa}} \cdot \mathrm{X}_{\mathrm{gk}}\right]$

$\mathrm{X}_{\text {esk }}=$ mole fraction eskolaite

$\mathrm{X}$ hem $=$ mole fraction hematite

Olivine-spinel Fe-Mg exchange thermometer :

$\mathrm{T}(\mathrm{K})=\left[15180+0.022 \cdot \mathrm{P}+(7000+0.011 \cdot \mathrm{P}) \cdot\left(1-2 \mathrm{X}_{\mathrm{fa}}\right)-2515 \cdot\left(1-2 \mathrm{XFe}_{\mathrm{Fp}}\right)\right.$ $\left.+15550 \cdot \mathrm{Y}_{\mathrm{Cr}}+20390 \cdot\left(\mathrm{YFe}_{\mathrm{Fe}}+\mathrm{Y}_{\mathrm{Ti}}\right)\right] /\left[\mathrm{R} \cdot \ln \mathrm{K}_{\mathrm{d}}+9.953\right]$

$\mathrm{K}_{\mathrm{d}}=\left[\mathrm{X}_{\mathrm{fo}} \bullet \mathrm{XFe}-\mathrm{sp}\right] /\left[\mathrm{X}_{\mathrm{fa}} \bullet \mathrm{XMg}-\mathrm{sp}\right]$

$\mathrm{XFe}-\mathrm{sp}=\mathrm{Fe} 2 /(\mathrm{Mg}+\mathrm{Fe} 2)$

$\mathrm{YCr}=\mathrm{Cr} /(\mathrm{Cr}+\mathrm{Al}+\mathrm{Fe} 3+2 \mathrm{Ti})$

$\mathrm{YTi}=2 \mathrm{Ti} /(\mathrm{Cr}+\mathrm{Al}+\mathrm{Fe} 3+2 \mathrm{Ti})$

\section{Ilmenite-Olivine oxygen sensor:}

$\Delta \log \left(\mathrm{fO}_{2}\right)^{\mathrm{FMQ}}=1611 / \mathrm{T}+1.123-0.057 \cdot(\mathrm{P} / \mathrm{T})+2 \cdot \log \left(\mathrm{aSiO}_{2}\right)+2 \cdot \log \left(\mathrm{X}_{\text {hem }}\right)$ $-(2098 / \mathrm{T}) \cdot \mathrm{X}_{\mathrm{gk}} \cdot \mathrm{X}_{\mathrm{ilm}}-(3876 / \mathrm{T}) \cdot \mathrm{X}_{\mathrm{esk}}+(3410 / \mathrm{T}) \cdot \mathrm{X}_{\mathrm{hem}}$

$(\mathrm{P}$ in bars, $\mathrm{T}$ in $\mathrm{K}) \quad-4 \cdot \log \left(1-\mathrm{X}_{\mathrm{fo}}\right)-(2826 / \mathrm{T}) \cdot\left(\mathrm{X}_{\mathrm{fo}}\right)^{2}$

Spinel-Olivine oxygen sensor:

$\Delta \log \left(\mathrm{fO}_{2}\right)^{\mathrm{FMQ}}=4.426-1895 / \mathrm{T}-0.037 \cdot(\mathrm{P} / \mathrm{T})+3 \cdot \log \left(\mathrm{aSiO}_{2}\right)-6 \cdot \log \left(1-\mathrm{X}_{\mathrm{fo}}\right)$

$-(4239 / \mathrm{T}) \cdot\left(\mathrm{X}_{\mathrm{fo}}\right)^{2}+2 \cdot \log \left(\mathrm{X}_{\mathrm{Fe}-\mathrm{sp}}\right)+4 \cdot \log \left(\mathrm{Y}_{\mathrm{Fe} 3}\right)$

( $\mathrm{P}$ in bars, $\mathrm{T}$ in $\mathrm{K})$

$+(4270 / \mathrm{T}) \cdot\left(\mathrm{Y} \mathrm{Al}_{\mathrm{Al}}\right)^{2}+(4626 / \mathrm{T}) \cdot\left(\mathrm{YTi}_{\mathrm{T}}\right)^{2}$

Silica activity expression (best fit) for peridotitic assemblages:

$\log \left(\mathrm{aSiO}_{2}\right)^{\text {olv-opx }}=-15.68 / \mathrm{T}-0.165-0.0087 \cdot(\mathrm{P} / \mathrm{T})+2 \cdot \log \left(\mathrm{X}_{\mathrm{en}}\right)-2 \cdot \log \left(\mathrm{X}_{\mathrm{fo}}\right)$ - $(1413 / \mathrm{T}) \cdot\left(1-\mathrm{X}_{\mathrm{fo}}\right)^{2}$

where $\mathrm{X}_{\mathrm{en}}$ is calculated from experimental $\mathrm{Fe}-\mathrm{Mg}$ partitioning relationships as follows: $X_{\text {en }}=A \cdot e^{B} /\left(1+A \cdot e^{B}\right)$ where $A=X_{\text {fo }} /\left(1-X_{f o}\right)$ and $B=134 / T-0.078+0.0027 \cdot(P / T)$. 